\title{
El relieve monumental de la diosa Tlaltecuhtli del Templo Mayor: estudio para la estabilización de su policromía
}

\author{
María Barajas Rocha
}

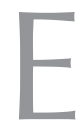
I 2 de octubre de 2006, el Programa de Arqueología Urbana (PAU) ${ }^{1}$ del - INAH Ilevó a cabo, dentro del predio de las Ajaracas-Campanas (en las calles de Argentina y Guatemala, Centro Histórico de la ciudad de México), el descubrimiento del monolito mexica más grande conocido hasta la fecha (Figura 1).

A partir del minucioso registro arqueológico realizado, ahora se puede afirmar que la monumental pieza (de $4.17 \times 3.62 \times 0.83 \mathrm{~m}$ y, aproximadamente, 12 toneladas) fue colocada por primera ocasión al pie de la pirámide de Templo Mayor, sobre el piso de su sexta etapa constructiva, atribuida al emperador mexica Ahuízotl. La escultura representa a la deidad Tlaltecuhtli en su advocación femenina.

A raíz de su hallazgo, en marzo del 2007 inició la séptima temporada del Proyecto Templo Mayor-INAH, dirigida por el doctor Leonardo López Luján, encaminada a explorar e investigar el área en la que se encontró el relieve. Para ello, en noviembre del mismo año el monolito de Tlaltecuhtli fue levantado y trasladado al nivel de la calle de Argentina, en donde se resguardaría dentro de una caseta de multipanel que fungió como laboratorio de conservación durante poco más de dos años (Figura 2).

La intervención en el relieve de Tlaltecuhtli representó un gran reto para la disciplina de la conservación-restauración, no sólo debido a sus monumentales dimensiones, sino también a circunstancias materiales y logísticas particulares. Éstas se abordaron bajo una perspectiva integral que comprendió desde intervenciones in situ hasta labores de traslado y montaje en el museo de sitio (Barajas et al. 2011).

Uno de los principales desafíos para la conservación de la Tlaltecuhtli derivó de la gran cantidad de pigmento original sobre su superficie, que se conservó circunstancialmente desde la época prehispánica hasta nuestros días, un hecho que, asimismo, representaba una oportunidad única para profundizar en el estudio de la escultura mexica. De ahí que, para abordar las alternativas

${ }^{1}$ El Programa de Arqueología Urbana (PAU), establecido por el profesor Eduardo Matos Moctezuma, comprende el estudio del propio edificio de Templo Mayor, así como del área que antiguamente constituía el recinto ceremonial mexica. Actualmente es coordinado por el arqueólogo Raúl Barrera. 


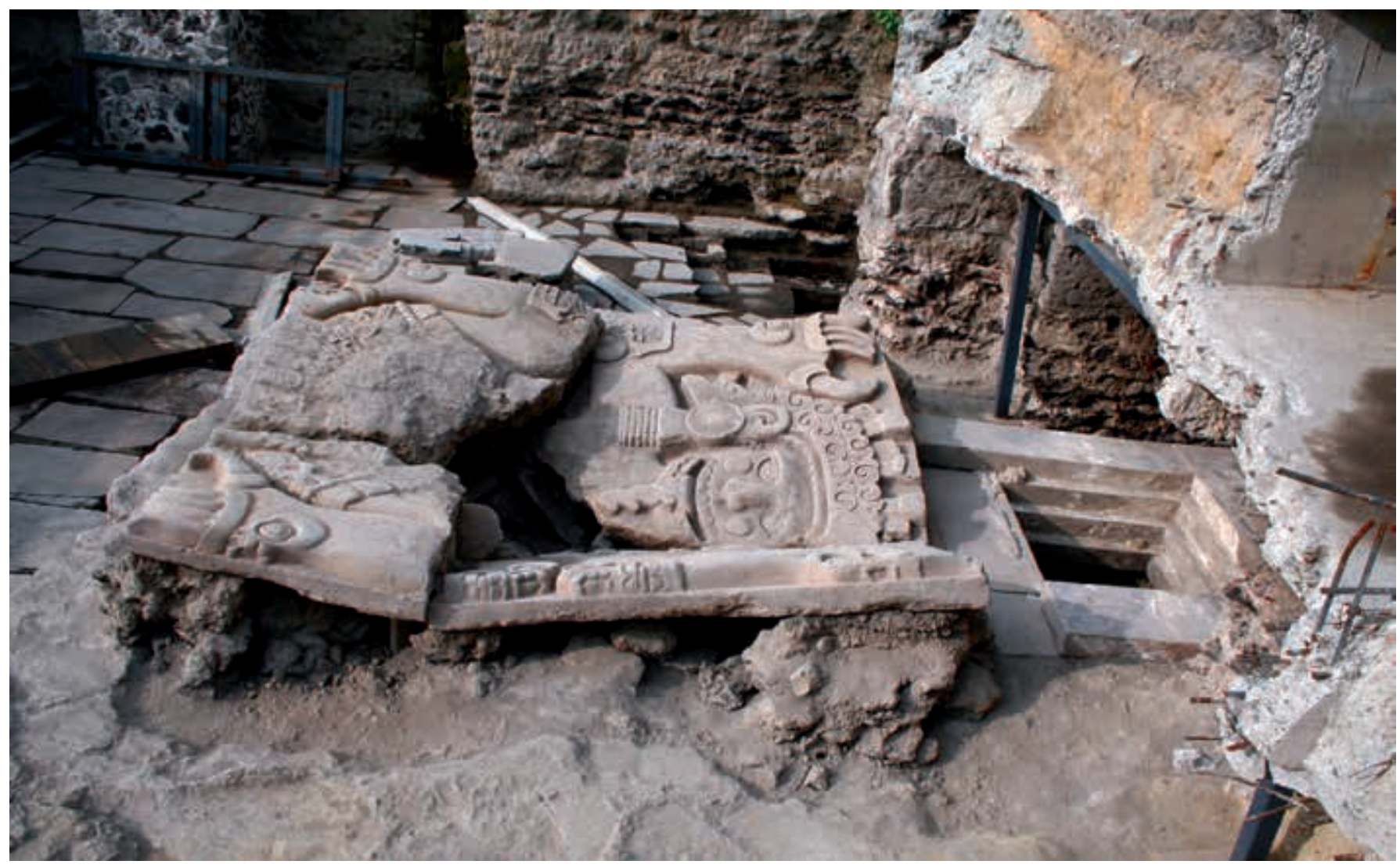

FIGURA 1. Vista general del relieve in situ (Cortesía: Proyecto Templo Mayor, INAH).

de intervención en materia de consolidación y fijado de la capa pictórica, se optara por una metodología de investigación que incluyera tecnologías de punta.

En este artículo se describe y analiza este interesante proceso de colaboración interdisciplinaria e interinstitucional, con el fin de dar a conocer tanto sus resultados como las posibles vertientes de trabajo futuro, derivadas de una experiencia única en el campo de la conservación arqueológica en México.

\section{El relieve}

Sabemos, por los estudios del geólogo Jaime Torres Trejo (2008: 3), que el monolito se labró en andesita de lamprobolita: los datos obtenidos mediante microscopia electrónica de barrido (SEM, por sus siglas en inglés) muestran claramente la presencia de óxidos de sílice como principal componente, así como de algunas trazas de aluminio, sodio, magnesio, potasio y un poco de hierro.

La pieza, cuya cara inferior es irregular, tiene forma rectangular. La talla, en la cara superior, realizada en alto relieve y mediante formas redondeadas con marcados volúmenes, muestra la imagen de un ser antropomorfo de cuerpo entero visto de frente.

El diseño de la imagen sigue una rigurosa simetría bilateral y, como ya se dijo, corresponde a la diosa mexica de la tierra: Tlaltecuhtli. La deidad Ileva los brazos abiertos,

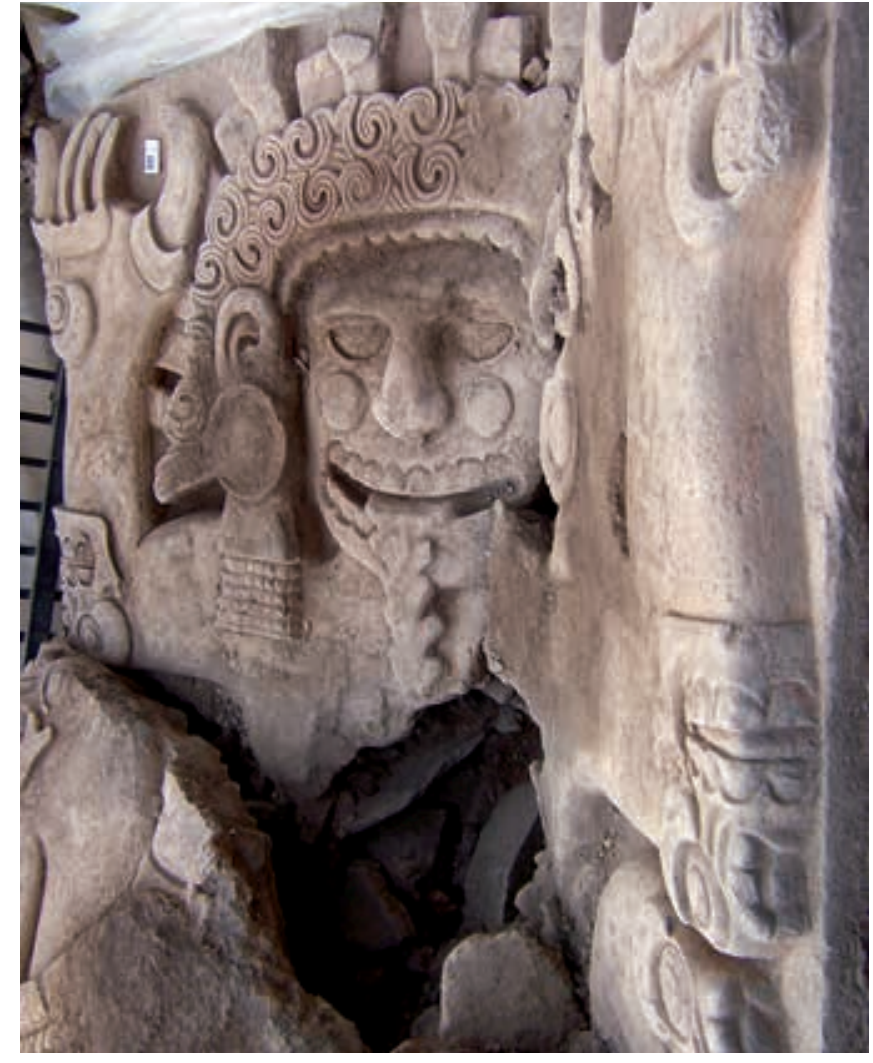

FIGURA 2. Relieve de Tlaltecuhtli durante el secado controlado (Cortesía: Proyecto Templo Mayor, INAH). 
doblados hacia arriba; sus piernas, desplegadas hacia afuera y flexionadas, guardan una postura de batracio o de alumbramiento (López Luján 2010: 77).

Presenta una cabellera rizada, cubierta de pigmento color rojo oscuro, propio de las divinidades de la noche y de la muerte, en la que aparecen insertadas banderas de papel que simbolizan el sacrificio. Sobre sus mejillas lleva discos redondos de color rojo, y mediante su boca semidescarnada sorbe un chorro de sangre proveniente de su abdomen. Porta grandes orejeras circulares.

El torso desnudo de la diosa muestra sus senos flácidos y pliegues a la altura del abdomen. En el centro, la escultura presenta un faltante, donde solamente se distingue el fragmento de un círculo rojo dentro del cual se observan dos pies calzados con sandalias. Viste una falda corta con el clásico motivo de estas deidades: cráneos humanos y huesos largos cruzados que se alternan. Sobrepuesta lleva una divisa dorsal o "falda de estrellas" (López Luján 2010: 87).

Desde el momento del hallazgo, cuando el relieve todavía estaba parcialmente cubierto de tierra, se observó que su superficie conservaba importante cantidad de restos de pigmento original. Después de los trabajos de limpieza y conservación, se constató que la paleta cromática utilizada en la decoración de esta escultura — limitada a los colores rojo, ocre, azul, blanco y negro (Figura 3) - es semejante a la detectada con anterioridad en otras esculturas y pinturas murales de la Zona Arqueológica Templo Mayor (López Luján 2005).

El análisis de la composición mineralógica de estos colores, hecho por Giacomo Chiari del Getty Conservation Institute (GCI) mediante difracción de rayos X (DRX), nos brindó los siguientes resultados (Figura 4):

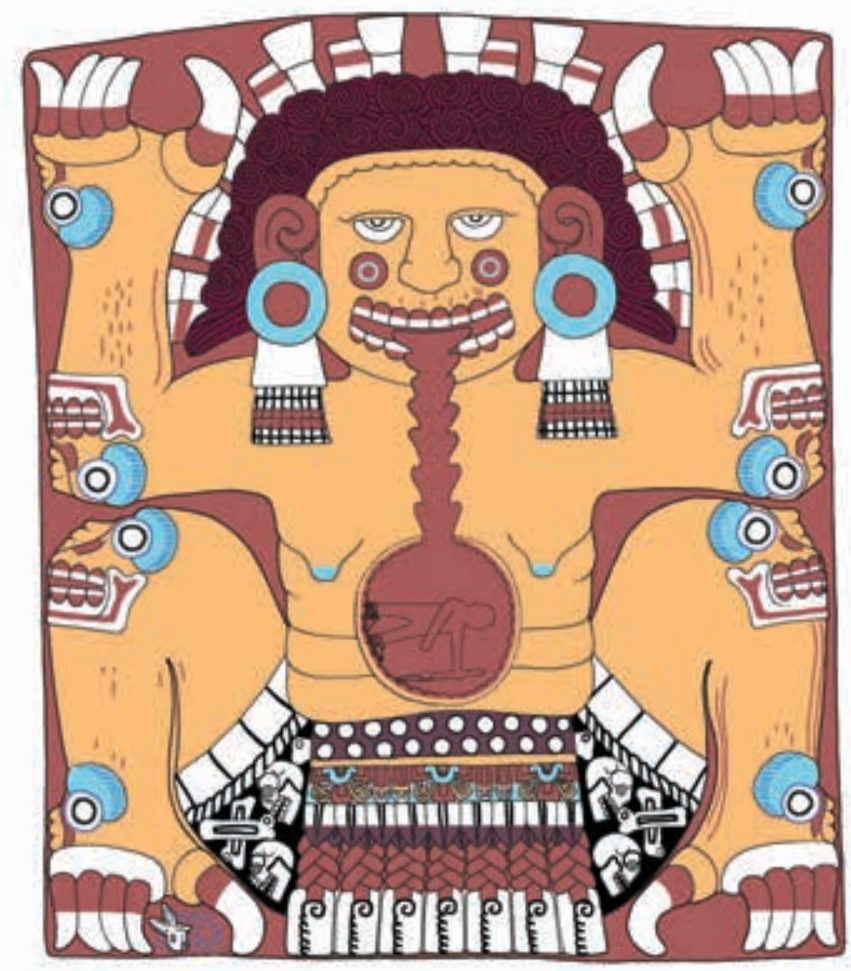

FIGURA 3. Dibujo reconstructivo del relieve y su policromía (Dibujo Julio Romero y Luz María Muñoz, 2009; cortesía: Archivo Proyecto Templo Mayor, INAH).

FIGURA 4. Tabla de resultados sobre composición mineralógica de los colores a partir de los análisis de Giacomo Chiari (Fuente Chiari 2008; cortesía: Archivo Proyecto Restauración Tlaltecuhtli, Templo Mayor, INAH).

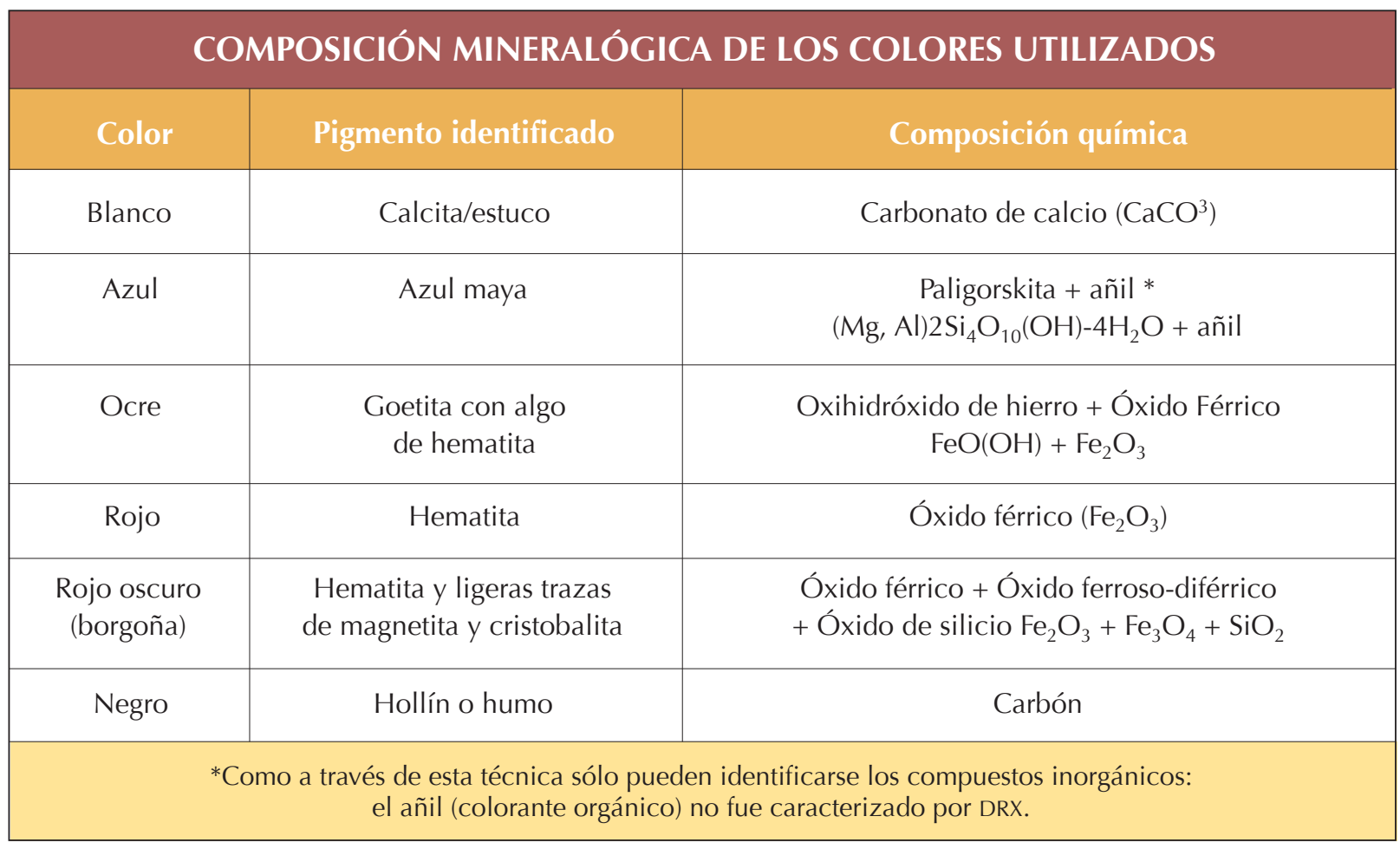




\begin{tabular}{|c|c|c|c|}
\hline \multicolumn{4}{|c|}{ RESULTADOS DE ANÁLISIS PARA IDENTIFICACIÓN DE AGLUTINANTES } \\
\hline Muestra & Proteínas - Aminoácidos & $\begin{array}{c}\text { Aceites grasos, ceras } \\
0 \text { resinas }\end{array}$ & Azúcares (exudados vegetales) \\
\hline 1. Blanco & $\begin{array}{l}0.2 \% \text { aminoácidos } \\
0.95 \% \text { gomas } \\
0.1 \% \text { lípidos }\end{array}$ & No se encontraron & $0.03 \%$ de azúcares (glucosa y manosa) \\
\hline 2. Azul maya & No se analizó & No se analizó & $<0.01 \%$ de azúcares \\
\hline 3. Ocre & $\begin{array}{l}0.2 \% \text { aminoácidos } \\
0.95 \% \text { gomas } \\
0.2 \% \text { lípidos }\end{array}$ & No se encontraron & $0.1 \%$ de azúcares (glucosa y manosa) \\
\hline 4. Rojo con ocre & No se analizó & No se analizó & 0.04\% de azúcares (glucosa y manosa) \\
\hline 5. Blanco & $\begin{array}{l}0.1 \% \text { aminoácidos } \\
0.89 \% \text { gomas } \\
0.1 \% \text { lípidos }\end{array}$ & No se encontraron & $0.02 \%$ de azúcares (glucosa y manosa) \\
\hline 6. Rojo & No se analizó & No se analizó & 0.01\% de azúcares (glucosa y manosa) \\
\hline 7. Blanco & No se analizó & No se analizó & $<0.01 \%$ de azúcares \\
\hline 10. Ocre & $\begin{array}{l}0.7 \% \text { aminoácidos } \\
0.95 \% \text { gomas } \\
0.9 \% \text { lípidos }\end{array}$ & No se encontraron & $<0.01 \%$ de azúcares \\
\hline
\end{tabular}

FIGURA 5. Resultados de los análisis para identificación de aglutinantes (Fuente Chiari 2008: 22; cortesía: Archivo Proyecto Templo Mayor, INAH).

Joy Mazurek, también del GCl, practicó una serie de análisis de cromatografía de gases acoplada a espectrometría de masas para saber si el aglutinante utilizado se elaboró con gomas vegetales, proteínas, aceites, ceras o resinas. A partir de su estudio, sólo pudieron detectarse bajísimas concentraciones de azúcar $(0.1 \%)$ - la glucosa y la manosa son los azúcares identificados consistentemente-, lo que hizo suponer, aunque sin poder afirmarlo todavía, que pudiera tratarse de un mucílago de orquídea. ${ }^{2}$ (Figura 5)

A partir de la observación bajo el microscopio óptico, en distintos cortes estratigráficos realizados a las muestras se notó que la mayoría de los pigmentos ${ }^{3}$ fue aplicada directamente sobre la superficie de la piedra. Es decir, se mezclaron con el aglutinante para disponerse directamente sobre el sustrato, lo que da como resultado, como menciona López Luján, superficies monocromáticas bien delimitadas, saturadas, opacas, uniformes y sin cambios de tonalidad ni sombras (Figuras 6a y 6b).

${ }^{2}$ Adhesivo de origen orgánico ampliamente empleado por los mexicas.

${ }^{3}$ A diferencia de los demás colores, el blanco, conformado por carbonato de calcio, se observó como una capa más gruesa y definida, lo cual era de suponer por tratarse de un recubrimiento de estuco; el negro, por su parte, se aplicó siempre sobre el estuco y no sobre la roca.
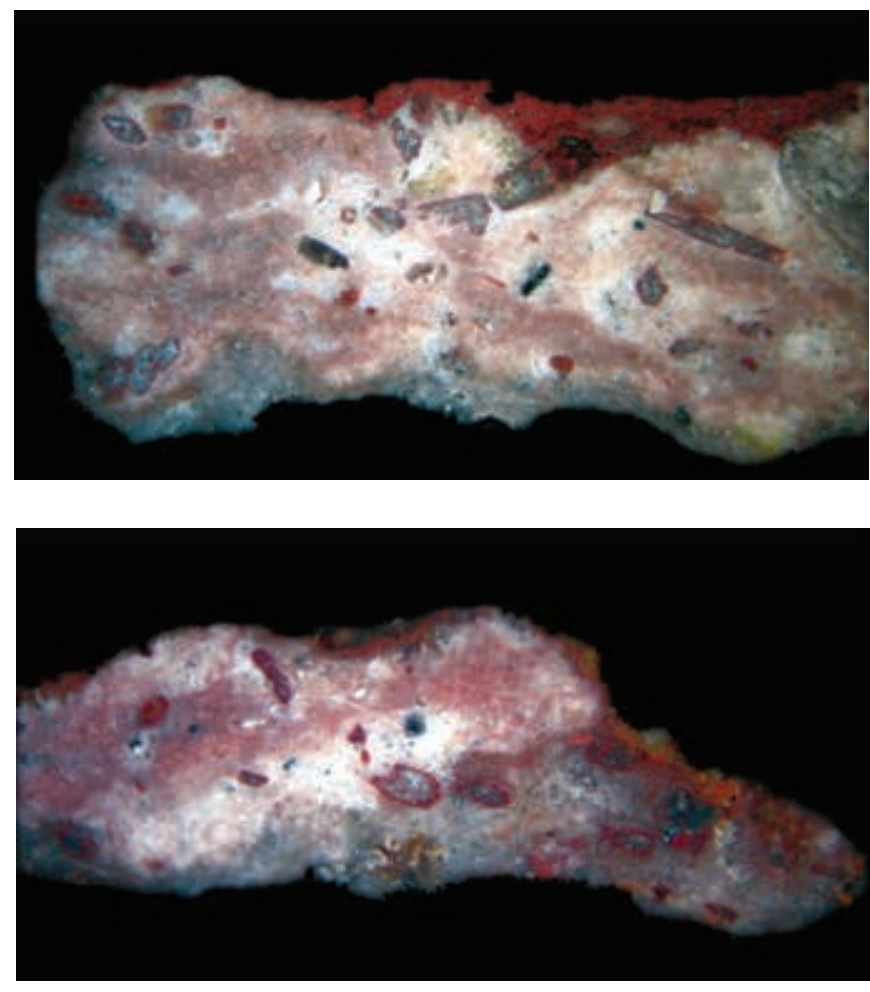

FIGURAS 6a y 6b. Cortes estratigráficos en los que se observa la película de pigmento aplicada directamente sobre el sustrato pétreo. Fotografías tomadas bajo el microscopio óptico (Fotografías Crisitina Barragán y Claudia Malváez; cortesía: Archivo Proyecto Restauración Tlaltecuhtli, Templo Mayor, INAH). 


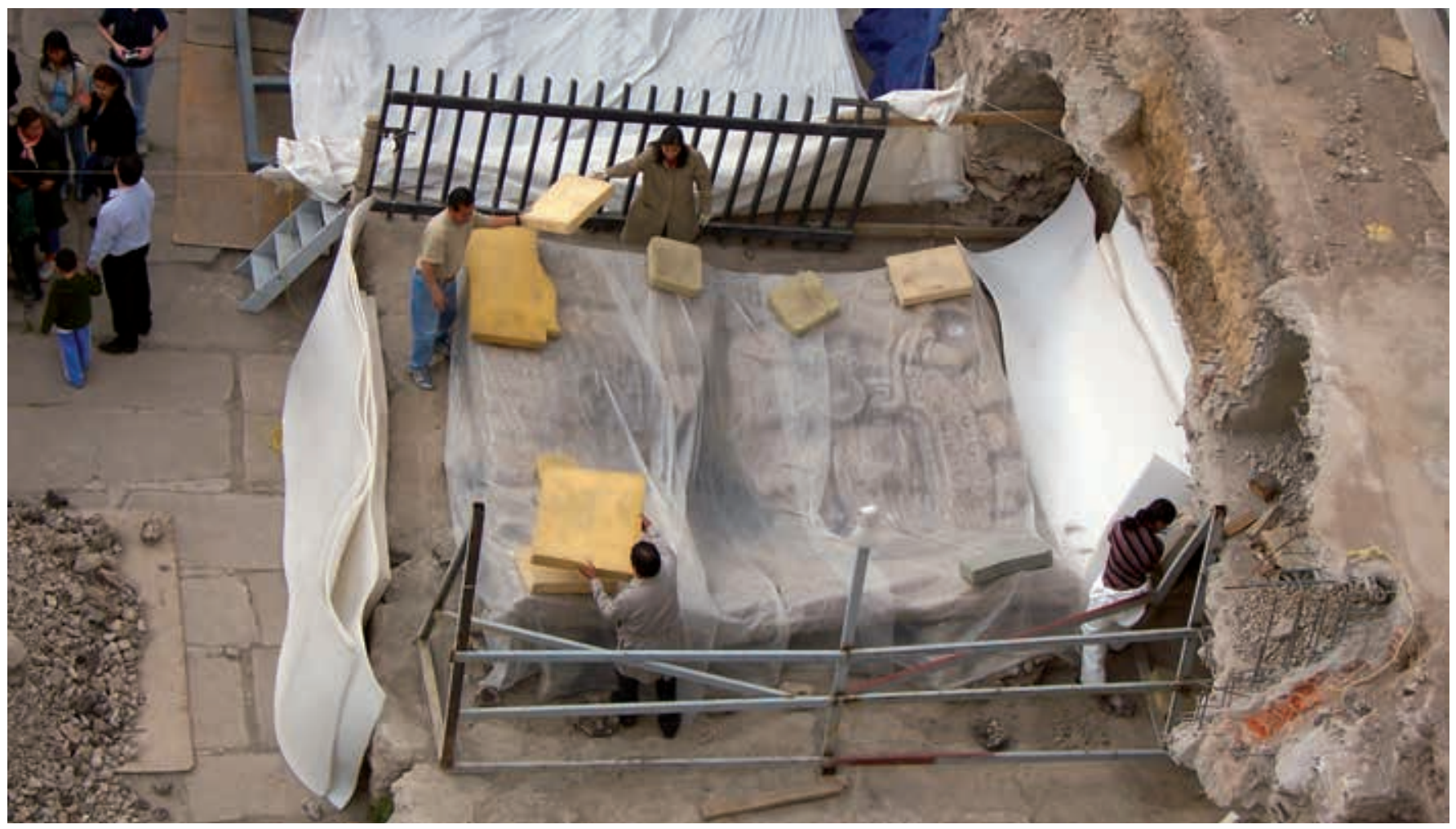

FIGURA 7. Control del proceso de secado in situ (Cortesía: Proyecto Templo Mayor, INAH).

\section{Estado de conservación}

La oportuna intervención, en el momento del hallazgo, del departamento de restauración de Templo Mayor, entonces dirigido por Virginia Pimentel (2006), sería determinante para la buena conservación de los materiales constitutivos del monolito. Desde su liberación se hizo lo necesario para tratar de evitar eventuales daños resultantes de los trabajos arqueológicos que se realizaban a su alrededor.

Dadas las características de humedad del subsuelo de la ciudad de México, y las condiciones climáticas preponderantes en los días inmediatos al descubrimiento, la piedra se encontró rodeada de tierra con un alto contenido de humedad, ante lo cual, y al observar que la escultura conservaba una importante cantidad de policromía original, se consideró primordial mantener lo más estable posible las condiciones de humedad relativa (HR) —que en el momento del hallazgo se reportó de 70\%-, lo cual se logró mientras se liberaba, y se evitó, así, un proceso de secado violento, lo cual hubiese provocado la contracción de los materiales constitutivos, con el riesgo de que posteriormente se desprendieran o perdieran (Pimentel 2006). Para esto el relieve se protegió con diversos materiales aislantes; se colocaron lonas plásticas para evitar la luz del sol directa, y la superficie pétrea se cubrió con materiales absorbentes previamente humedecidos con agua destilada (Figura 7).

Aunque la roca se observó estable desde el momento del hallazgo y aun después del proceso de secado, Torres
Trejo (2008: 48) identificó de manera constante en la matriz la presencia de vidrio volcánico, el cual en muchos casos manifestaba ya una devitrificación, además de cierta oxidación presente en los minerales ferromagnesianos como producto de la alteración.

Además de que estaba fragmentada en cuatro grandes pedazos, en la andesita en que fue tallado el monolito aparecían de manera localizada algunos efectos de deterioro, como disgregación superficial, producto de un posible proceso de intercambio de humedad constante hacia el interior y el exterior de la roca; erosión y desgaste, más marcados en la zona del faldellín de la diosa, probablemente derivados del uso que tuvo en época prehispánica; desprendimientos y faltantes, quizá originados a partir la estructura fluidal ${ }^{4}$ que presenta este tipo de rocas; grietas tal vez causadas por esfuerzos mecánicos en el momento en que se dieron las fracturas estructurales, y concreciones calcáreas y desportilladuras, generadas por roces o golpes.

En cuanto a su decoración, se pudo notar, ya avanzado el proceso de limpieza mecánica de la imagen, que la capa pictórica se había conservado casi en su totalidad. No obstante, dejaba ver un fuerte grado de pulverulencia, debido a la pérdida del aglutinante original.

En las áreas decoradas en colores azul maya y negro, se identificaron zonas con exfoliación, acaso debida a la

\footnotetext{
${ }^{4}$ Esta textura, típica en las rocas ígneas, se observa como líneas de distintas
} tonalidades, paralelas entre sí, y es producto de la cristalización del magma. 
pérdida de adherencia con el sustrato, y en las decoradas con estuco, craqueladuras y fisuras generadas por la pérdida de cohesión.

\section{Fijado de la capa pictórica}

$\mathrm{Al}$ encontrarnos frente a una escultura monumental casi totalmente policromada, el proceso de fijado de la capa pictórica sería, a nuestro modo de ver, el punto crucial en la intervención de restauración del monolito; por ello la decisión sobre cómo debíamos determinar el método más adecuado, representó un reto muy grande y, a la vez, emocionante.

Conscientes de que era necesario proceder a su fijado para contribuir a devolver estabilidad a la capa de policromía, también lo estábamos de que esta restitución implicaría la adición de algún material consolidante que necesariamente sería irreversible y modificaría las características físico-químicas del material original. Por esto se buscaron compuestos que, dependiendo de su naturaleza química e interacción con los materiales originales, pudieran presentar cierta estabilidad ante los factores microclimáticos y que, a su vez, permitieran futuros retratamientos o reaplicaciones. De ahí que nos resultara conveniente llevar a cabo un trabajo analítico dirigido al conocimiento de la interrelación del material constitutivo con las sustancias fijativas con las que habíamos de experimentar.

En consecuencia, y con la colaboración del doctor Pedro Bosch Giral y su equipo del Instituto de Investigaciones en Materiales de la Universidad Nacional Autónoma de México (IIM-UNAM), se realizó una investigación científica enfocada en el análisis, la comprensión y la valoración de las siguientes sustancias fijativas:

Paraloid $B 72^{\circledR}$ al $2.5 \%$ en xileno. Polímero sintético comúnmente utilizado en el campo de la restauración.

Funori al $1.25 \%$ en agua destilada, y baba de nopal 1:1 en agua destilada. Estos dos compuestos se seleccionaron por razón de la compatibilidad que guardan con el aglutinante original identificado.

Methocel y Klucel-G ${ }^{\oplus}$ al $1 \%$ en agua destilada. Compuestos derivados de la celulosa, que se caracterizan por ser bastante estables, además de medianamente reversibles (Barberá Durón 2004). ${ }^{5}$

KSE $300 E^{\circledR}$ 1:1 en alcohol. Etil éster de silicato fabricado por la marca Remmers, de origen alemán. Este compuesto es compatible con el sustrato pétreo.

\footnotetext{
${ }^{5}$ Barberá Durón ha analizado, específicamente para su uso en conservación de obras con soporte de papel, la estabilidad del Klucel-G en relación con el Methocel (Barberá Durón 2004).
}

Paralelamente a la selección de los posibles fijativos, se separaron seis fragmentos de la misma andesita constitutiva del monolito, a los que se les aplicó en seco, con ayuda de un pincel, una película de pigmento de color ocre (goetita). Posteriormente se aplicaron por goteo los diferentes fijativos preparados en laboratorio.

Los objetivos de los análisis fueron:

a) Conocer la composición y la morfología de la película que eventualmente se formaría en la superficie pétrea al aplicar el fijativo.

b) Conocer la variación cromática que podría llegar a tener la capa pictórica original, tanto a corto como a largo plazo, una vez aplicado el fijativo.

c) Comprender, a corto y mediano plazo, el comportamiento del sistema poroso existente en la andesita ya tratada.

\section{Metodología de análisis}

Se sometió un grupo de seis muestras, más una muestra piloto sin fijativo, a una cámara de envejecimiento acelerado, operada por el químico Miguel Ángel Canseco, del IIM. Dentro de la cámara, que tiene variaciones de humedad, temperatura y exposición a rayos UV, se alternaron repetidamente, hasta completar las veinticuatro horas del día, ${ }^{6}$ ocho horas a $50{ }^{\circ} \mathrm{C}$ con radiación ultravioleta y HR entre $80-100 \%, y$, posteriormente, cuatro horas de entre $44-50{ }^{\circ} \mathrm{C}$ en condensación a $100 \%$ de HR.

Al realizar los análisis las muestras envejecidas se compararon con un grupo de muestras sin envejecer.

Asimismo, con la técnica DRX se pudo caracterizar el material mineral, y se identificaron fases cristalinas, comparando además los difractogramas de las muestras antes y después de su envejecimiento.

\section{Resultados}

Además de que en las muestras se encontraron los patrones relacionados con la composición de la andesita (albita, ortoclasa) y de la película de pigmento (goetitahidróxido de hierro), se puede decir que en las muestras tratadas con paraloid y methocel no se observaron diferencias significativas. Después del envejecimiento, los picos correspondientes a la goetita se volvieron, en el caso del funori, más intensos - lo que sugiere una posible aglomeración de las partículas del pigmento-y en el de la baba del nopal, por el contrario, son menos definidos - lo que apunta a una posible fragmentación en las partículas de pigmento, producto del envejecimiento o de una posible disolución del hidróxido de hierro en el polisacárido-. En cuanto al KSE 300 E, la fase

\footnotetext{
${ }^{6}$ Las muestras se dejaron dentro de la cámara durante 66.66 días, con lo que se obtuvo un envejecimiento equivalente a 32 meses: 600 horas dentro de la cámara, esto es, 25 días equivalen a un año de tiempo real.
} 
cristalina correspondiente a la formación de pequeñas partículas de cuarzo desapareció tras el envejecimiento -lo que hace suponer que se da una redistribución del compuesto-, mientras que, después de ese proceso, la muestra tratada con Klucel presentó nuevos compuestos cristalinos.

Respecto de los análisis, los hechos por la observación mediante SEM hicieron posible comprender las características en cuanto a la textura y la morfología en superficie; adicionalmente, se efectuaron análisis elementales, mediante microanálisis de rayos $X$ por dispersión de energías, sobre algunas zonas seleccionadas en las muestras. En las imágenes se pudo observar que el funori induce la formación de grandes aglomerados con bordes afilados, mientras que el Klucel forma una película que cubre la superficie de la roca, a manera de aglomerados globulares. Después del envejecimiento, en ambas muestras se detectó una superficie con mucho más textura, producto, probablemente, de la degradación de la película de fijativo.

En el caso de la baba de nopal y el Methocel, las partículas de goetita se observaron con mayor textura antes de ser intemperizadas. Ambos fijativos parecen formar una capa que sella la porosidad, la cual se pierde con el tiempo como producto de la biodegradación.

En las muestras tratadas tanto con paraloid como con KSE 300 E, se observó: 1) en la superficie de una y otra, un recubrimiento que dejaba algunas zonas libres de pigmento; 2) que, con el primer producto, se formó una película lisa y homogénea, mientras que el segundo cubrió — pero, aun así, permitió observar- zonas globulares, sin que la muestra estuviera completamente sellada, y 3) a través del envejecimiento, una capa compacta y más gruesa en el caso de la muestra con paraloid, y, en el de la tratada con KSE $300 \mathrm{E}$, una superficie menos uniforme y menos compacta, que deja notar pequeños espacios y textura.

Encaminado a evaluar las modificaciones cromáticas, a corto y a mediano plazo, de cada una de las sustancias estudiadas en relación tanto con el sustrato pétreo como con la película de pigmento, con el apoyo del profesor Manlio Favio Salinas Nolasco (ENCRyM-INAH) se realizó un análisis de colorimetría y cambios cromáticos que consistió en hacer mediciones espectrofotométricas sobre cada una de las muestras antes y después del proceso de intemperismo. Sus resultados nos llevan a afirmar que en general todos los consolidantes modificaron los colores originales de los componentes, desplazando en cierto grado el color de la capa pictórica (naranja amarillento) con un salto en la escala de Munsell (naranja amarillo). Además, Salinas Nolasco concluyó respecto de la direccionalidad de los cambios cromáticos presentados antes y después del envejecimiento que la baba de nopal y el

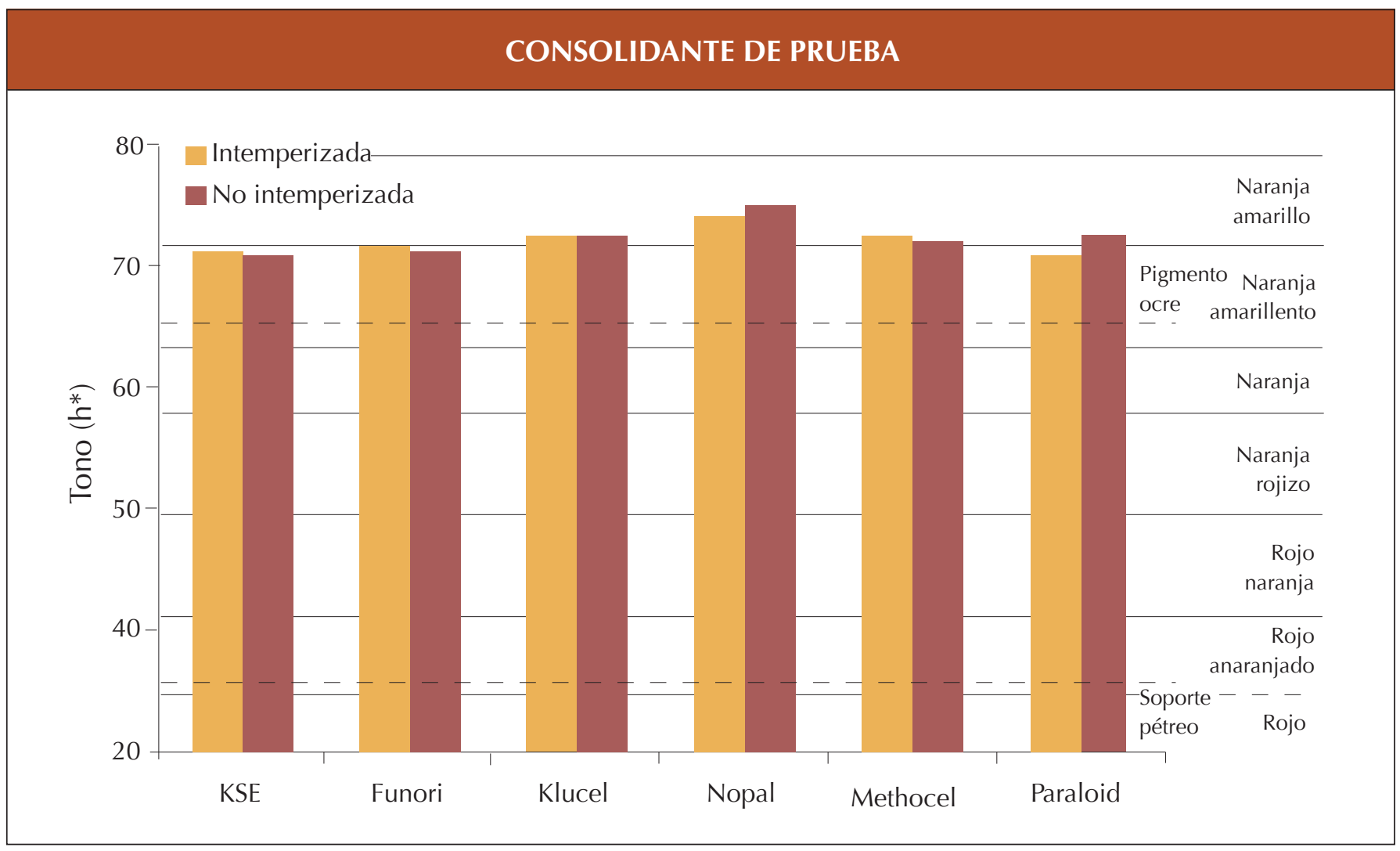

FIGURA 8. Magnitudes absolutas de los valores de tono para cada consolidante, tabla elaborada por Manlio Favio Salinas Nolasco (Fuente Salinas Nolasco, 2009: 19; cortesía: Archivo Proyecto Restauración Tlaltecuhtli, Templo Mayor, INAH). 


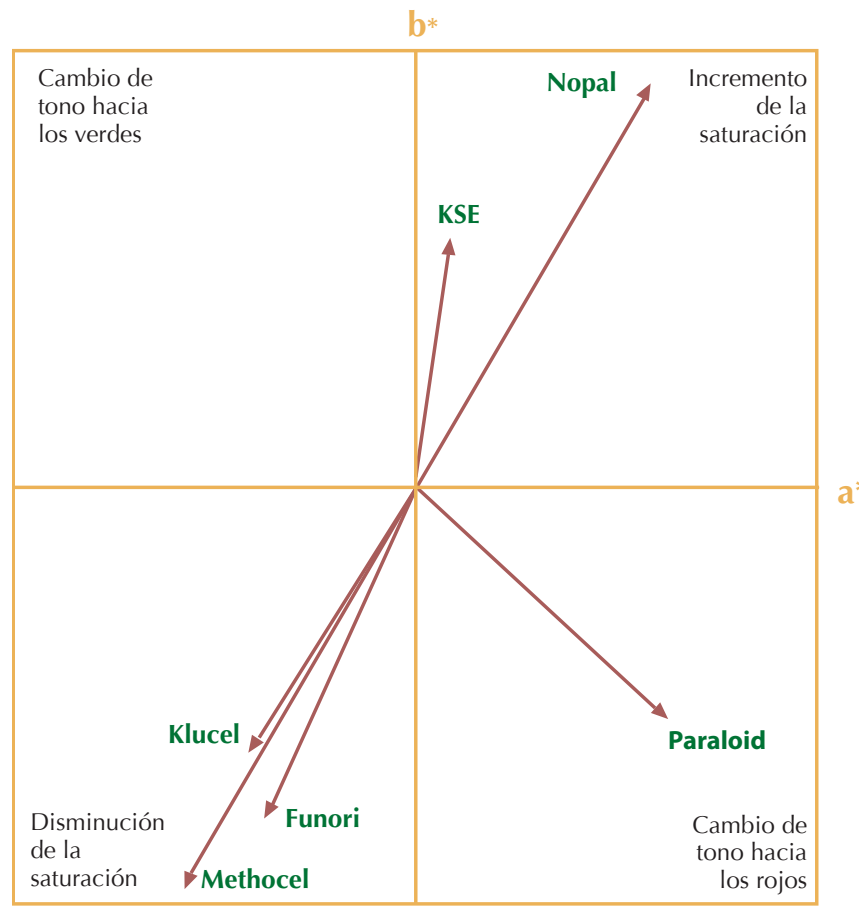

FIGURA 9. Direcciones clasificadas de acuerdo con el sentido del cambio de color después del intemperismo (Fuente Salinas Nolasco, 2009: 13; cortesía: Archivo Proyecto Restauración Tlaltecuhtli, Templo Mayor, INAH).

KSE 300 E contribuyeron a incrementar la saturación del color, mientras que el Klucel, el Funori y el Methocel la disminuyeron, en tanto que el Paraloid modificó el color hacia los rojos.

Pruebas físicas. Una vez obtenidos estos resultados de investigación, se decidió evaluar, mediante una sencilla prueba, los seis fijativos analizados, con el fin de reducir nuestro campo de opciones para llevar a cabo el fijado definitivo de los pigmentos. Aunque algunos de éstos son acuosos, se decidió rodar un hisopo humedecido en agua destilada sobre la superficie de las muestras ya tratadas, considerando que los compuestos, una vez que se aplicaran y reaccionaran, formarían una película que no necesariamente sería soluble en agua, además de que se tomaría en cuenta la resistencia a la fricción generada durante la prueba. En lo que toca al desprendimiento de pigmento: no lo hubo con el paraloid ni con el KSE 300 E; fue mínimo con el funori y el Klucel, y con la baba de nopal y con el Methocel, el algodón presentó una importante cantidad de pigmento desprendido.

A partir de lo anterior, se resolvió seguir trabajando únicamente con el Paraloid y el KSE 300 E, pues hasta el momento ambos habían logrado fijar por completo el pigmento a la superficie pétrea.

Estudio de porosidad: adsorción de nitrógeno. A partir de nuestra inquietud sobre el comportamiento que podría tener la película formada sobre la superficie del relieve,

\begin{tabular}{|c|c|c|}
\hline \multicolumn{3}{|c|}{$\begin{array}{r}\text { RESULTADOS ANÁLISIS POR AI } \\
\text { DE NITRÓGENO }\end{array}$} \\
\hline Muestra & $A^{+}, m^{2} \cdot g-1$ & $d^{++}, \AA$ \\
\hline Andesita & 18.5 & 174 \\
\hline Andesita + pintura & 20.7 & 122 \\
\hline Paraloid nuevo & 10.0 & 324 \\
\hline Paraloid viejo & 10.8 & 247 \\
\hline KSE nuevo & 21.2 & 125 \\
\hline KSE viejo & 23.3 & 172 \\
\hline \multicolumn{3}{|c|}{$\begin{array}{l}\text { † Área superficial medida por el método de BET. } \\
\text { Debido a que la superficie específica es una propiedad que depende de la técnica } \\
\text { utilizada para medirla, el método BET (Brunnauer, Emmett y Teller) es reconocido } \\
\text { mundialmente como estándar. La determinación de la adsorción de nitrógeno } \\
\text { requiere un equipo capaz de medir volumétricamente la cantidad de moléculas } \\
\text { absorbidas por el sólido en análisis. }\end{array}$} \\
\hline $\begin{array}{l}\text { "Diámetro de poro promedio est } \\
\text { Este método (Barret, Joyer y Haler } \\
\text { en varios intervalos: el valor med } \\
\text { espesor de la capa absorbida y el }\end{array}$ & $\begin{array}{l}\text { or el método BJH } \\
\text { siste básicamente e } \\
\text { esión entre cada un } \\
\text { edio de los poros. }\end{array}$ & $\begin{array}{l}\text { isoterma } \\
\text { calcular el }\end{array}$ \\
\hline
\end{tabular}

FIGURA 10. Resultados análisis por adsorción de nitrógeno, tabla elaborada por el doctor Jorge Balmaseda Era del IIM-UNAM (Fuente Barajas et al. 2011: 114; cortesía: Archivo Proyecto Restauración Tlaltecuhtli, Templo Mayor, INAH).

nos fue sugerida la posibilidad de realizar un análisis por adsorción de nitrógeno, técnica analítica útil para determinar el área superficial total de un sólido tomando en cuenta la porosidad de material, el tamaño de poro y su distribución. Dicho estudio nos permitiría conocer tanto el área superficial como la distribución y los diámetros de los poros y, con ello, una vez fijada la capa pictórica, comprender el patrón de porosidad. En la Figura 10 se presentan los resultados obtenidos.

A partir de estos resultados se concluyó, como puede observarse en la tabla anterior, que el paraloid crea una película que sella los poros de menor tamaño, generando valores mayores en el diámetro promedio, y que el KSE $300 \mathrm{E}$, por su parte, mantiene el diámetro promedio del poro similar al que tenía la roca antes de aplicar el fijativo. Lo anterior puede deberse a que este consolidante, al hacer contacto con la superficie porosa de la roca, forma una fase intermedia de uniones de cristales en forma de sílice, que no llegan a obstruir o tapar por completo los poros.

\section{Definición y puesta en marcha del proceso de fijado}

Una vez concluidos los análisis de adsorción de nitrógeno, consideramos que ya contábamos con evidencia 
de que KSE 300 E reaccionaría de manera adecuada, a corto y a mediano plazo, tanto con la roca como con la película de pigmento, por lo que teníamos bases sólidas sobre las cuales encaminar nuestra propuesta de intervención hacia la aplicación de un fijativo compatible con el material original. Se escogió como método de aplicación el de la aspersión, con el fin de que no hubiera demasiada penetración hacia la roca y el efecto consolidante se diera únicamente a nivel superficial.

Es importante recalcar que este producto reacciona tanto con la humedad del ambiente como con la presente al interior de los poros de la piedra, mediante una separación de dióxido de silicio (acuoso y amorfo), el cual se enlaza a través de segmentos blandos y ocasiona que el gel de sílice actúe como cementante. La velocidad de separación del gel va relacionada con la temperatura y la humedad.

A partir de las consideraciones anteriores, se buscó controlar las condiciones circundantes, y las aplicaciones se llevaron a cabo con una HR promedio de aproximadamente $40-50 \%$. El proceso de fijado se inició en el mes de febrero de 2010, siempre con las ventanas y las puertas de la caseta cerradas, con el fin de lograr un mayor control.

En la preparación y la aplicación del consolidante se tomaron las medidas de seguridad necesarias para los restauradores que las realizaron, como el uso de guantes, la protección de ojos con lentes - ya que los componentes del KSE 300 E pueden llegar a causar problemas en córneas-y la utilización de mascarilla con filtros de protección ante vapores orgánicos.

Dentro de la caseta-laboratorio se llevaron a cabo cuatro aplicaciones, dejando un lapso de aproximadamente $2 \frac{1}{1} 2$ semanas entre una y otra, en cada uno de los fragmentos. Después de la última aplicación, hecha el 19 de abril de 2010, los pigmentos estaban relativamente estables, lo que nos permitió comenzar a planear las maniobras de traslado al interior del Museo del Templo Mayor y montaje de los cuatro fragmentos del relieve, las cuales tendrían lugar el 17 de mayo de 2010 (Figura 11).

El relieve ya colocado y exhibido en el interior del museo se revisó detalladamente, con el fin de evaluar el resultado del proceso de fijado, y a partir de éste decidimos que sería necesario programar dos aplicaciones más sobre toda la superficie de la escultura: la primera realizada en agosto y la segunda en diciembre de 2010.

Además del monitoreo permanente de las condiciones microclimáticas circundantes y de la revisión periódica

\footnotetext{
${ }^{7}$ Dado que el proceso de fijado requiere cierto periodo de tiempo para que la reacción se lleve a cabo entre cada aplicación, el trabajo en la caseta-laboratorio se enfocó en lograr estabilizar la capa pictórica para que ésta resistiera durante el traslado de los fragmentos al interior del Museo del Templo Mayor. Posteriormente, con la escultura ya montada en el recinto, se evaluaría el proceso de fijado y, de ser necesario, se llevarían a cabo más aplicaciones de fijativo.
}

tanto de la estabilidad como de la decoración de la escultura, el departamento de restauración del Museo / Proyecto Templo Mayor ha efectuado cotidianamente diversas labores de mantenimiento y preservación: los días lunes se realiza, con la ayuda de una aspiradora de mochila, una limpieza superficial en la base museográfica alrededor del relieve; cada tres o cuatro meses, la superficie polícroma del monolito se somete a un proceso de limpieza superficial general: sobre un andamio tubular que permite tener acceso a todos los puntos de la escultura, se va retirando, con brochas de pelo suave y perillas —además de que se tiene la aspiradora encendida a un lado-, la capa de polvo depositada (Figura 12).

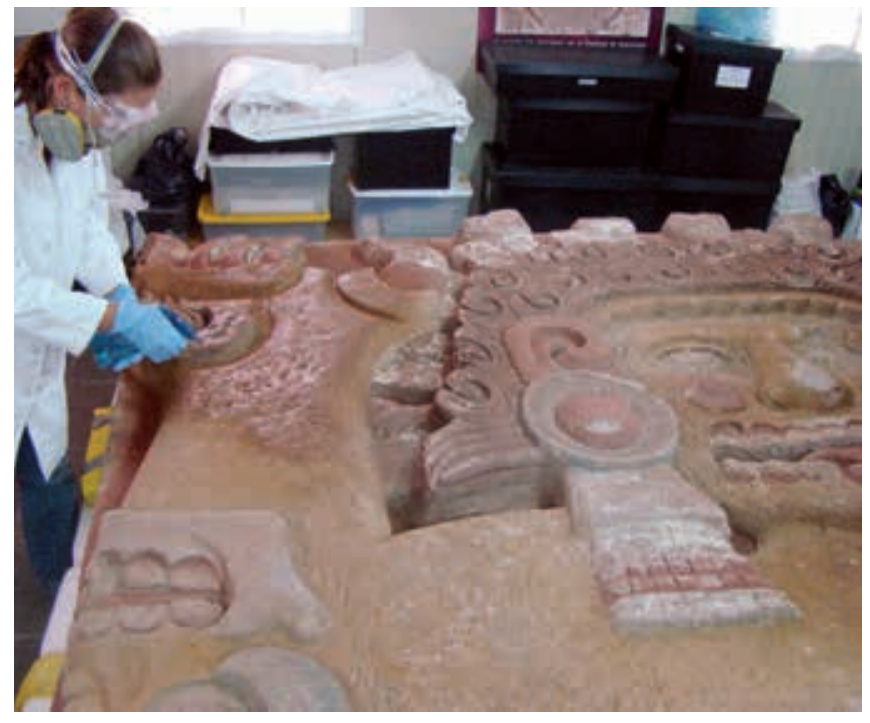

FIGURA 11. Proceso de fijado de los pigmentos en la caseta-laboratorio de conservación (Fotografía Claudia Malváez, 2010; cortesía: Archivo Proyecto Restauración Tlaltecuhtli, Templo Mayor, INAH).

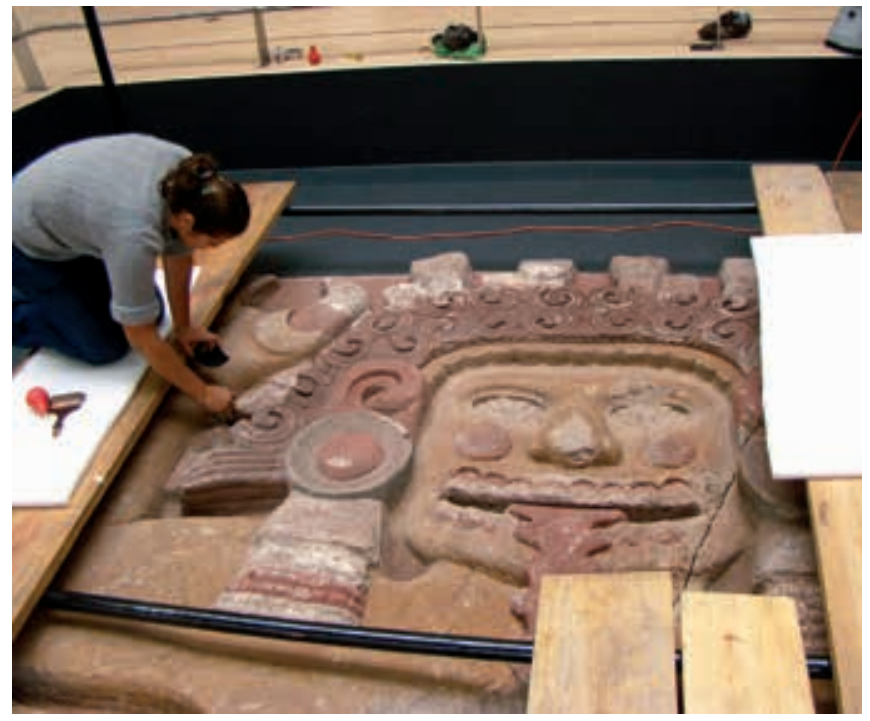

FIGURA 12. Trabajos periódicos de revisión y mantenimiento del relieve y su policromía (Cortesía: Archivo Proyecto Restauración Tlaltecuhtli, Templo Mayor, INAH). 


\section{Consideraciones finales}

Como hemos comentado, el proyecto de restauración del monolito que representa a la diosa Tlaltecuhtli ha implicado, desde el momento de su liberación hasta su montaje temporal dentro del Museo del Templo Mayor, y su posible reubicación en el sitio preciso donde fue hallado, una larga serie de discusiones, propuestas, y procesos de intervención y planeación.

En este artículo nos hemos abocado específicamente a la problemática del fijado de los pigmentos (color) a la superficie, siendo ésta la primera vez que se lleva a cabo una investigación científica tan amplia antes de definir las estrategias de intervención para la conservación de esta monumental escultura. Los resultados de esta investigación y lo aprendido a través de la propia intervención contribuirán, creemos, a generar nuevas propuestas y procedimientos sobre el tratamiento de otros materiales pétreos policromados, $y$, en general, al avance dentro de la disciplina de la conservación arqueológica.

Durante el desarrollo de esta intervención, fueron notablemente enriquecedoras las discusiones sobre problemáticas específicas entre quienes nos encargamos directamente de la restauración y los arqueólogos, físicos, geólogos, arquitectos e ingenieros asociados al proyecto, proceso interdisciplinario que ayudó a respaldar académicamente nuestras decisiones y acciones. Las discusiones de este tipo suelen ser muy difíciles, ya que cada uno de los participantes utilizamos, según nuestra especialidad, lenguajes distintos, y centramos la atención en diferentes aspectos del problema. Así como esto puede ser enriquecedor, si no se tiene la experiencia necesaria se puede caer en un trabajo desarticulado, en el cual se coleccionan datos producidos por diferentes áreas sin llegar al punto de vincularlos unos con otros.

Los estudios y análisis realizados, encaminados a sustentar la formulación de nuestra propuesta de intervención, permitieron que nos centráramos en acciones específicas; constatando la importancia de llevar a cabo este tipo de ejercicios y valoraciones previas. Ahora es necesario continuar, a mediano y largo plazo, con la evaluación del desempeño del consolidante bajo situaciones reales de envejecimiento natural.

Es así como la experiencia generada a lo largo de este trabajo intenta constituir una contribución al desarrollo de la disciplina, y, además, abrir nuevas líneas específicas, tanto de acción como de investigación, para otros casos futuros.

\section{Referencias}

Barajas, María 2011 "Informe de la restauración y exhibición del relieve monumental de la diosa Tlaltecuhtli Templo Mayor", mecanoescrito, Archivo del Museo del Templo Mayor, Museo del Templo Mayor-INAH.

Barajas, María, Pedro Bosch, Claudia Malváez, Cristina Barragán y Enrique Lima

2010 "Stabilization of the Tlaltecuhtli Monolith Pigments", Journal of Archaeological Science, 37, 2881-2886.

Barberá Durón, Natalia Valeria

2004 "Metilcelulosa e hidroxipropilcelulosa; estudio comparativo de su estabilidad y características de envejecimiento", tesis de licenciatura, México, ENCRyM-INAH.

Barragán, Cristina y Claudia Malváez 2010 “Evaluación del paraloid B72 y KSE 300 E como fijativos para los pigmentos presentes en el monolito de la diosa Tlaltecuhtli del Templo Mayor", tesis de licenciatura, México ENCRYM-INAH.

Chiari, Giacomo

2008 "Results of the X-Ray Diffraction Analysis of Samples of the Earth Goddess Tlaltecuhtli", manuscrito, The Getty Conservation Insititute.

López Luján, Leonardo

2010 Tlaltecuhtli, México, Fundación Conmemoraciones / INAH.

López Luján, Leonardo, Giacomo Chiari, Alfredo López Austin y Fernando Carrizosa

2005 "Línea y color en Tenochtitlan. Escultura policromada y pintura mural en el recinto sagrado de la capital mexica", Estudios de Cultura Náhuatl, 36, 15-45.

Pimentel, Virginia

2006 "Anteproyecto de intervención para la conservación y restauración del material pétreo policromado en proceso de excavación, PAU, Conjunto Ajaracas", mecanoescrito, Archivo del Museo del Templo Mayor.

Salinas Nolasco, Manlio Favio 2009 "Evaluación de los cambios cromáticos y ópticos de seis consolidantes sobre muestras de prueba del monolito Tlaltecuhtli", mecanoescrito, Archivo del Museo del Templo Mayor, ENCRYM-INAH.

Torres Trejo, Jaime

2008 Petrografía y procedencia probable de algunos materiales constructivos y escultóricos del Templo Mayor de MéxicoTenochtitlan, mecanoescrito. 


\section{Resumen}

En octubre de 2006 se llevó a cabo el descubrimiento de un monolito de grandes dimensiones frente al recinto del Templo Mayor que representa a la diosa mexica de la Tierra: Tlaltecuhtli. En colaboración con diversos especialistas e instituciones nacionales y extranjeras, los restauradores encargados de su conservación realizaron una extensa investigación científica, cuya finalidad consistió en encontrar la mejor opción para lograr fijar la película de pigmentos que coloreaba la superficie pétrea, que los llevó a comprender el comportamiento de seis diferentes sustancias. Este artículo muestra la importancia del trabajo interdisciplinario $-\mathrm{y}$ de contar con el apoyo de diversos especialistas-, en el que los procesos de conservación estén fundamentados en la investigación y la valoración previa para determinar la manera más adecuada de abordar la problemática.

\section{Palabras clave}

Fijado de pigmentos, escultura monumental mexica, investigación, Tlaltecuhtli.

\section{Abstract}

In October 2006 a colossal monolith, representing the Earth goddess Tlaltecuhtli, was discovered in front of the Templo Mayor Archaeological Site in Mexico City. In collaboration with different national and international specialists and institutions, the restorers from the Templo Mayor carried out extensive scientific research in order to find the best option to fix and stabilise the pigments that colour the stone, leading them to understand the behavior of six different substances. This article shows the importance of having the support of diverse specialists and of interdisciplinary work, in which conservation processes are based on research and previous assessments to determine the best way of solving a conservation problem.

\section{Keywords}

Pigment fixation, Aztec monumental sculpture, research, Tlaltecuhtli.

Título en inglés: "The monumental sculpture of the goddess, Tlaltecuhtli, in the Templo Mayor Archaeological Site: study to stabilise the sculpture's polychromy" 